\title{
Drug reaction with eosinophilia and systemic symptoms in a child on multiple antiepileptics
}

\author{
Jyotindra Narayan Goswami ${ }^{1}$, Pankaj C. Vaidya ${ }^{1}$, Arushi Gahlot Saini ${ }^{1}$, Dipankar De ${ }^{2}$, \\ Bishan Dass Radotra ${ }^{3}$, Pratibha D. Singhi ${ }^{1,4}$ \\ Departments of ${ }^{1}$ Pediatrics, ${ }^{2}$ Dermatology, Leprology and Venereology, and ${ }^{3}$ Histopathology; ${ }^{4}$ Division of Pediatric Neurology, \\ Post Graduate Institute of Medical Education and Research, Chandigarh, India. \\ E-mail: doctorpratibhasinghi@gmail.com \\ Received: 4th October 2016, Revised: 2nd January 2017, Accepted: 19th April 2017
}

SUMMARY: Goswami JN, Vaidya PC, Saini AG, De D, Radotra BD, Singhi PD. Drug reaction with eosinophilia and systemic symptoms in a child on multiple antiepileptics. Turk J Pediatr 2017; 59: 197-199.

Drug reaction with eosinophilia and systemic symptoms (DRESS) is an adverse drug-reaction that may mimic systemic illnesses and have a fulminant presentation. We describe an 8-year-old girl with epilepsy and exposure to multiple anti-epileptics who presented with fever, extensive maculopapular rash, cervical lymphadenopathy, hepatomegaly, progressive anemia and transaminitis. Infections, autoimmune disorders and hematological or reticuloendothelial malignancies were excluded. Based on the proposed diagnostic criteria, a diagnosis of DRESS was concluded. Her skin biopsy showed atypical findings consistent with erythema multiforme. Suspected anti-epileptic drugs were discontinued. She was administered pulse methyl-prednisolone therapy and broad-spectrum antibiotics along with adequate supportive management. Unfortunately, the child succumbed to nosocomial sepsis. Our case highlights the importance of early suspicion for diagnosis of pediatric DRESS, avoidance of polytherapy and institution of early immunomodulation to improve the outcomes in children in this condition.

Key words: drug rash, drug reaction, DRESS.

Drug reaction with eosinophilia and systemic symptoms (DRESS) is an adverse drug reaction that may mimic systemic illnesses and have a fulminant presentation. Numerous drugs have been reported to cause DRESS among which antiepileptic drugs are common offenders, especially when used as polytherapy. ${ }^{1}$ High index of suspicion in the appropriate clinical context remains the cornerstone for diagnosis of DRESS in children and timely institution of immunomodulation for therapy.

We describe an 8-year-old girl with idiopathic generalized epilepsy and DRESS secondary to multiple anti-epileptic drug exposure who presented with fever, maculopapular rash, cervical lymphadenopathy, hepatomegaly, anemia and transaminitis. She was administered pulse methyl-prednisolone therapy but succumbed to nosocomial infection. Our case underscores the importance of early suspicion and diagnosis of pediatric DRESS, avoidance of polytherapy and institution of early immunomodulation to improve outcomes in children.

\section{Case Report}

An 8-year-old girl presented with high grade, remittent fever for the past two months followed by acute-onset, generalized rash for the past three days. There was no associated alteration in sensorium, headache, vomiting, double vision, neck-pain, focal motor deficits, ear-discharge, respiratory symptoms, jaundice, abdominal distension, burning micturition, joint pains, chronic discharging sinus or bleeding manifestations. She had been diagnosed with idiopathic generalized epilepsy at the age of 5 years and despite anti-epileptic therapy continued to have generalized tonic-clonic seizures once every 4-6 months. She had been initiated on multiple anti-epileptic drugs (sodium valproate up to $30 \mathrm{mg} / \mathrm{kg} /$ day, oxcarbazepine $21 \mathrm{mg} / \mathrm{kg} /$ day, levetiracetam $47 \mathrm{mg} / \mathrm{kg} /$ day, 
phenobarbitone $3 \mathrm{mg} / \mathrm{kg} /$ day and clobazam $0.2 \mathrm{mg} / \mathrm{kg} /$ day) for seizure recurrences. Her perinatal history and development were normal. She performed averagely in school and had minor behavioral problems. Family history was not contributory.

At admission, her temperature was $40^{\circ} \mathrm{C}$, heart rate $132 / \mathrm{min}$, respiratory rate $30 /$ min and blood pressure was 114/72 $\mathrm{mmHg}$. Examination showed normal sensorium, pallor, submandibular lymphadenopathy, mild hepatomegaly (span $11 \mathrm{~cm}$ ) and a generalized erythematous, blancheable, confluent, maculopapular rash over face, trunk, extremities (including palms and soles) occupying nearly $60 \%$ of her body surface area. There was no exfoliation, bleeding or discharge from the rash and mucosal surfaces were not involved. Rest of the systemic examination was normal. She was investigated for systemic infections, autoimmune reticuloendothelial malignancies and DRESS. Phenobarbitone, valproate and oxcarbazepine were withheld. Ceftriaxone (100 mg/kg/day divided 12 hourly) and oral doxycycline $(2 \mathrm{mg} / \mathrm{kg} /$ day $)$ were empirically started.

Laboratory investigations showed hemoglobin $6.9 \mathrm{~g} / \mathrm{dl}$, total leucocyte count $12,600 / \mathrm{mm}^{3}$, absolute eosinophil count $4,790 / \mathrm{mm}^{3}$, (normal 500 to $2,500 / \mathrm{mm}^{3}$ ), aspartate transaminase 207 $\mathrm{U} / \mathrm{L}$ and alanine transaminase $148 \mathrm{U} / \mathrm{L}$ (normal $<40 \mathrm{U} / \mathrm{L}$ ). Blood and throat swab cultures, anti-

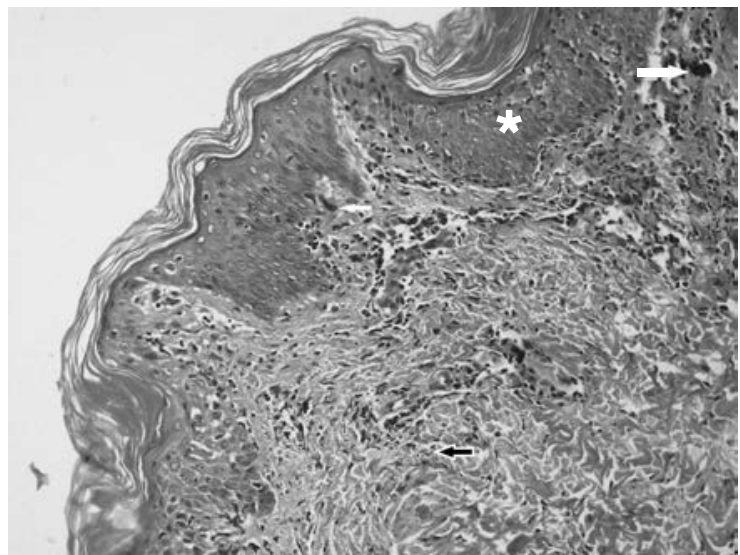

Fig 1. Figure showing high power microscopy of skin biopsy of the patient.

The figure portrays orthokeratosis, follicular plugging, lymphocytic exocytosis, epidermal spongiosis (depicted by white star) and apoptotickeratinocytes (depicted by white arrow) with moderately dense mononuclear infiltrate (black arrow) in upper dermis
streptolysin-O titre, Widal, Mantoux intradermal skin test, sputum for acid-fast bacilli, serological tests for dengue, mycoplasma, scrub typhus, brucella, cytomegalovirus, herpes, EpsteinBarr, parvo, human immunodeficiency virus, toxoplasma and hydatid were negative. Serum antinuclear antibody titers, galactomannan and immunoglobulin levels were normal. Cerebrospinal fluid analysis and magnetic resonance imaging of brain were normal. Fineneedle aspiration cytology from cervical lymph node revealed reactive hyperplasia. Skin biopsy showed atypical features for DRESS (Fig. 1 $1)$. Bone-marrow examination did not show features of active infection or malignancy. A final diagnosis of DRESS was concluded based on the proposed diagnostic criteria ${ }^{3}$ and she was administered pulse methyl prednisolone (30 mg/kg/day for 3 days) followed by oral corticosteroids. However, she succumbed to nosocomial infection.

Informed consent was obtained from the child's parents prior to submission of the above case report for publication.

\section{Discussion}

Our case emphasizes the importance of early diagnosis of pediatric DRESS, prompt withdrawal of offending anti-epileptic polytherapy and timely institution of immunomodulation to improve the outcomes in children. Clinical features may be pleomorphic and skin biopsy may be non-specific in DRESS; hence, a high clinical suspicion and exclusion of infections is needed in children.

DRESS is a clinical diagnosis based on Registry of Severe Cutaneous Adverse Reactions (RegiSCAR) study group criteria ${ }^{3}$ : fever (temperature $>38.5^{\circ} \mathrm{C}$ ), lymphadenopathy, eosinophilia, atypical lymphocytosis, rash, organ involvement, recovery after more than 15 days of drug stoppage and exclusion of other etiologies. Our child scored more than five on the RegiSCAR criteria indicating "definite DRESS". Patient-tailored investigations are primarily needed to exclude infections, autoimmune disorders and reticuloendothelial malignancies which may mimic the symptoms of DRESS and to look for multisystem involvement as in the index case. ${ }^{2}$

Aromatic anti-epileptic drugs (phenytoin, phenobarbitone, and carbamazepine) have 
been implicated in nearly one-third of the drugs causing DRESS. ${ }^{1}$ Even non-aromatic anti-epileptic drugs such as oxcarbazepine, zonisamide, felbamate and lamotrigine may cause DRESS and also demonstrate crossreactivity with aromatic anti-epileptic drugs in $40-80 \%$ cases. $^{2}$ Exposure to one or many of these drugs possibly triggered DRESS in our index patient. Besides drugs, reactivated viruses such as human herpes virus 6 and 7, EpsteinBarr and cytomegalovirus have also been implicated. However, serological investigations in our patient were negative for these viruses.

Skin biopsy findings are variable in DRESS and may range from the common superficial perivascular lymphocytosis and necrotic keratinocytes to the rare dermal eosinophilic infiltration, interface dermatitis, erythema multiform or acute generalized erythematous pustulosis.1,3,5 Atypical features such as erythema multiform in our patient may delay the correct diagnosis. ${ }^{3}$

As the pathogenesis of DRESS involves type IV hypersensitivity reaction with severe multi-systemic immune dysregulation, ${ }^{4}$ its management comprises of discontinuation of triggering drugs and institution of aggressive immunomodulation such as corticosteroids, intravenous immunoglobulin and plasmapheresis. , $^{2,7,8}$

There is growing evidence of genetic factors triggering adverse drug reactions. ${ }^{9}$ HLA-B* ${ }^{*}$ 15:02 has been proven to commonly cause carbamazepine induced rash in Han Chinese population. ${ }^{10}$ Some other known genetic predispositions for adverse drug reactions include HLA-A*31:01 with carbamazepine, HLA-B*57:01, HLA-DR7 and HLA-DQ3 with abacavir and HLA-B*58:01 with allopurinol. ${ }^{9}$ However genetic testing in DRESS is not standardized as of date.

We used pulse methyl prednisolone therapy followed by oral corticosteroids in the index patient; intravenous immunoglobulin could not be administered due to financial constraints. However, the patient succumbed to nosocomial infections.

To summarize, our case highlights that DRESS may be a great masquerader in the pediatric age group and needs high index of suspicion for diagnosis, especially in children exposed to multiple anti-epileptic drugs. Discontinuation of offending drugs, early immunomodulation and aggressive supportive care are its core management strategies.

\section{REFERENCES}

1. Cacoub P, Musette P, Descamps V, et al. The DRESS syndrome: A literature review. Am J Med 2011; 124: 588-597.

2. Criado PR, Criado RF, Avancini JM, Santi CG. Drug reaction with eosinophilia and systemic symptoms (DRESS) / Drug-induced hypersensitivity syndrome (DIHS): A review of current concepts. An Bras Dermatol 2012; 87: 435-449.

3. Kardaun SH, Sidoroff A, Valeyrie-Allanore L, et al Variability in the clinical pattern of cutaneous sideeffects of drugs with systemic symptoms: does a DRESS syndrome really exist? Br J Dermatol 2007; 156: 609-611.

4. Hall DJ, Fromm JS. Drug reaction with eosinophilia and systemic symptoms syndrome in a patient taking phenytoin and levetiracetam: a case report. J Med Case Reports 2013; 7: 2-3.

5. Choudhary S, McLeod M, Torchia D, Romanelli P. Drug Reaction with eosinophilia and systemic symptoms (DRESS) syndrome. J Clin Aesthetic Dermatol 2013; 6: 31-37.

6. Creamer D, Walsh SA, Dziewulski P, et al. UK guidelines for the management of Stevens-Johnson syndrome/toxic epidermal necrolysis in adults 2016. J Plast Reconstr Aesthetic Surg 2016; 69: 119-153.

7. Solensky R,Khan DA,Bernstein IL,et al. Drug Allergy: An Updated Practice Parameter Endorsed by: American Academy of Allergy, Asthma and Immunology, the American College of Allergy, Asthma and Immunology, and the Joint Council of Allergy, Asthma and Immunology. Ann Allergy Asthma Immunol 2010; 105: 259-273.

8. Descamps V, Ben Saïd B, Sassolas B, et al. Management of drug reaction with eosinophilia and systemic symptoms (DRESS). Ann Dermatol Vénéréologie 2010; 137: 703-708.

9. Daly AK. Pharmacogenomics of adverse drug reactions. Genome Med 2013; 5: 5-6.

10. Chung WH, Hung SI, Hong HS, et al. Medical genetics: a marker for Stevens-Johnson syndrome. Nature 2004; 428: 486. 